\title{
Design and Implementation of Sliding Mode Controller using Coefficient Diagram Method for a nonlinear process
}

\author{
R.Selvaraj ${ }^{1}$, S.Abraham Lincon ${ }^{2}$, R.Satheesh $\mathrm{Babu}^{3}$ \\ ${ }^{I}$ (Assistant Professor, Electronics \& Instrumentation Engg, Annamalai University, India) \\ ${ }^{2}$ (Professor, Electronics \& Instrumentation Engg, Annamalai University, India) \\ ${ }^{3}$ (PG Scholar, Electronics \& Instrumentation Engg, Annamalai University, India)
}

\begin{abstract}
The method proposed in this paper uses a second order Sliding Mode Control Algorithm(SMC), is designed and implemented for a non linear process which makes use of the Coefficient Diagram Method(CDM) technique to find the controller parameters. The proposed controller is inherently 2 degree of freedom structure. This work considers the liquid level control of a spherical tank system. Simulation of the process model has been carried out by Mat lab Simulink software. Along with simulation results, real time responses of spherical tank liquid level control process also presented for evaluation and validation of proposed method.
\end{abstract}

Keywords: Adaptive control, Second order SMC, 2 Degree of freedom, SMC-CDM, Spherical tank level process.

\section{INTRODUCTION}

The Sliding Mode Control approach is recognized as one of the efficient tools to design robust controllers for complex high-order nonlinear dynamic plant operating under uncertainty conditions. The major advantage of sliding mode is its low sensitivity to plant parameter variations and disturbances which eliminates the necessity of exact modeling. Sliding mode control enables the decoupling of the overall system motion into independent partial components of lower dimension and, as a result, reduces the complexity of feedback design. The basic properties are the order reduction, invariance principle and its effectiveness towards chattering in real time applications make it more popular. The SMC approach consists of two steps:

1) The first step is the choice of a manifold in the state space such that, once the state trajectory is constrained on it, the controlled plant exhibits the desired performance.

2) The second step is represented by the design of a discontinuous state-feedback capable of forcing the system state to reach, in finite time, such a manifold (accordingly called "SLIDING MANIFOLD").

In this work, the design and implementation of SMC using Coefficient diagram method is presented to improve standard designs in adaptive control schemes. Section 2 describes the design of SMC algorithm for SISO plants. Section 3 is devoted to the development of 2 degree of freedom SMC using CDM for SISO plants. Section 4 presents the results and analysis of performance comparison of controllers. The conclusion is presented in section 5 .

\section{SECOND ORder SLIDING Mode CONTROL Algorithm}

Consider an uncertain SISO nonlinear system described by the vector differential equation

$$
\dot{x}=\emptyset(t, x(t))+\gamma(t, x(t)) u(t)
$$

Which is affine in the control $u$ and where $x \in X \subset \Re^{n}$ is a state vector, $u \in U \subset \Re$ is a bounded input and $t$ is the independent time variable. Select a sliding surface as follows,

$$
s=S(t, x)
$$

Such that by zeroing it, the control objective is achieved. Further assume that the sliding surface $s$, has relative degree two with respect to the control input i.e. $\frac{\partial}{\partial x} S(t ; x)(t ; x)=0$. Thus the system dynamics can be written in the following form

$$
\ddot{s}=f(t, s, \dot{s}, x)+g(t, s, \dot{s}, x) u
$$

The dynamics in equation (2) are assumed to satisfy the following bounding conditions $0<G_{\min } \leq g(t, s, \dot{s})$ $\leq G_{\max }$ and $|f(t, s, \dot{s})| \leq F ;|s| \leq s_{0}$. Where $G_{\min }, G_{\max }, s_{0}$ and $F$ are some positive constants. Given the physical limits of most practical engineering systems, the assumed bounding conditions are not unduly restrictive.

A Second order sliding control algorithm is applied to stabilize the dynamics without the knowledge of the derivative of the sliding variable $(\dot{s})$. The algorithm is given as follows

$$
u(t)=-\lambda \operatorname{sign}(s)+u_{2}(t)
$$




$$
\dot{u_{2}}=\left\{\begin{aligned}
-k u, & |u|>u_{0} \\
-W \operatorname{sign}(s), & |u| \leq u_{0}
\end{aligned}\right.
$$

The corresponding sufficient conditions for finite time convergence are

$$
u_{0}=\frac{F}{G_{\min }} ; \lambda>u_{0} ; \quad \mathrm{k}, W>0
$$

\section{2 DEGREE OF FREEDOM ADAPTIVE SMC USING CDM TECHNIQUE}

The proposed method which is used to design a sliding mode controller satisfying the required time and frequency domain specifications consists of two steps. In the first step, a method is proposed to compute the global and the local stability regions using the stability boundary locus approach. In the second step, the CDM method is used to design SMC controllers for which the step responses have a required overshoot and an acceptable settling time. As a result of combining these two steps, the Frequency and Time Domain Plot(FTDP) map is obtained. The FTDP map, which is a graphical tool, shows the relation between the stabilizing parameters of the SMC controller and the chosen frequency and time domain performance criteria on the same $\lambda, u_{2}$ plane. Thus, one can choose a SMC controller providing all of the desired GM, PM, MO, and $t_{s}$ specification values together.

A general schema of the 2DOF control system is shown in Fig.1.

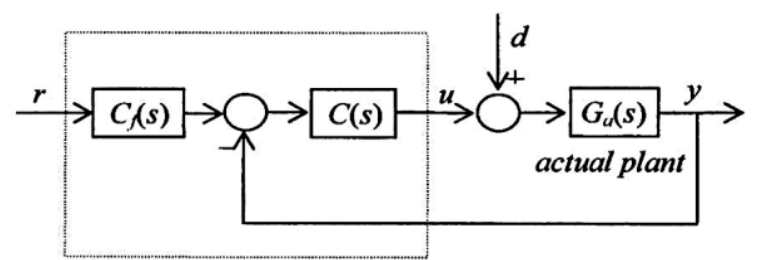

Fig. 1. A 2DOF control system structure.

Where

$$
G_{a}(s)=\mathrm{G}(\mathrm{s}) e^{-\theta s}=\frac{N(s)}{D(s)} e^{-\theta s}
$$

The closed-loop characteristic polynomial $P(s)$ of the system, i.e., the numerator of $1+C(s) G_{a}(s)$, can be written as

$$
P(s)=s D(s)+\left(\lambda \operatorname{sign}(s) s+u_{2}\right) N(s) e^{-\theta s}
$$

where all or some of the coefficients $a_{i}, i=0,1,2, \ldots, n$ are the function of $\lambda, \mathrm{u}_{2}$, and $e^{-\theta s}$ depending on the order of $N(s)$, and $D(s)$ polynomials. The characteristic polynomial is given in the following form

$$
P(s)=a_{n} s^{n}+\cdots+a_{1} s+a_{0} \quad=\sum_{i=0}^{n} a_{i} s^{i}
$$

The stability index $\gamma_{i}$, the equivalent time constant $\tau$, and stability limit $\gamma_{i}^{*}$ are defined as follows.

$$
\begin{aligned}
& \gamma_{i}=\frac{a_{i}^{2}}{a_{i+1} a_{i-1}}, \quad i=1 \sim n-1 \\
& \tau=\frac{a_{1}}{a_{0}} \\
& \gamma_{i}^{*}=\frac{1}{\gamma_{i+1}}+\frac{1}{\gamma_{i-1}}, \quad \gamma_{n}=\gamma_{0}=\infty
\end{aligned}
$$

From these equations the following relations are derived.

$$
a_{i}=\frac{\frac{a_{i+1}}{a_{i}}=\frac{\frac{a_{j}}{a_{j-1}}}{\gamma_{i} \gamma_{i-1} \cdots \gamma_{j+1} \gamma_{j}}}{a_{0} \tau^{i}{ }^{i-1} \gamma_{i-2} \cdots \gamma_{2}^{i-2} \gamma_{1}^{i-1}} \quad i \geq j
$$

Then characteristic polynomial will be expressed by $a_{0}, \tau$ and $\gamma_{i}$ as follows.

$$
P(s)=a_{0}\left[\left\{\sum_{i=2}^{n}\left(\prod_{j=1}^{i-1} \frac{1}{\gamma_{i-j}^{j}}\right)(\tau s)^{i}\right\}+\tau s+1\right]
$$

When the performance specifications are given, usually the rise time, the settling time, the overshoot, and the peak time are used for the time response specification. However from the CDM design point of view, only the settling time $t_{s}$ is meaningful, because it gives upper bound of $\tau$, where $t_{s}=2.5 \sim 3 \tau$. The frequency response specifications are used for the high frequency attenuation characteristics and the low frequency disturbance rejection characteristics. 


\section{Results And Analysis}

The designed SMC is implemented in real time also compared with a PI controller. The set point is given in terms of percentage of level. $20 \%$ of level is given as nominal operating value, after $14000(\mathrm{~s})$ the set point has been changed to $30 \%$. The load is applied at the valve in outlet, for 10 liter/min change in outflow. For the sampling time, $1 \mathrm{sec}$ is selected.

Table 1. Plant parameters

\begin{tabular}{|l|c|}
\hline \multicolumn{1}{|c|}{ Process variables } & Nominal operating conditions \\
\hline Level $(\mathrm{h})$ & $1 \mathrm{~m}$ \\
\hline Flow rate, $\left(\mathrm{F}_{\text {in }}\right)$ & $0.2215 \mathrm{~m}^{2} / \mathrm{sec}$ \\
\hline Radius of the tank $(\mathrm{r})$ & $1 \mathrm{~m}$ \\
\hline Constant of the outlet valve $\left(\mathrm{c}_{\mathrm{s}}\right)$ & $0.05 \mathrm{~m}^{2}$ \\
\hline Outlet valve stem position $\left(\mathrm{x}_{\mathrm{s}}\right)$ & 1 \\
\hline Gravitational acceleration $(\mathrm{g})$ & $9.8 \mathrm{~ms}^{-1}$ \\
\hline Maximum level & $2 \mathrm{~m}$ \\
\hline
\end{tabular}

Table 1 provides the description of Spherical tank parameters where table 2 shows the PI controller parameters for various linearized plant models as different operating levels $10 \%, 50 \%$ and $66 \%$ of tank level. Table 3 provides the controller settings for SMC-CDM.

Table 2. PI controller parameters(ZN method) concerning plant models

\begin{tabular}{|c|c|c|c|}
\hline Linearized models & Transfer function models & \multicolumn{2}{|c|}{ PI Controller parameters } \\
\cline { 3 - 4 } & & $\mathrm{K}_{\mathrm{c}}$ & $\mathrm{K}_{\mathrm{i}}$ \\
\hline Model 1 & $\frac{4 e^{-120 s}}{440 s+1}$ & 0.825 & 0.002 \\
\hline Model 2 & $\frac{6 e^{-130 s}}{1200 s+1}$ & 1.385 & 0.003 \\
\hline Model 3 & $\frac{2.75 e^{-150 s}}{1050 s+1}$ & 2.29 & 0.005 \\
\hline
\end{tabular}

Table 3. SMC-CDM controller parameters for simulation

\begin{tabular}{|c|c|c|c|c|}
\hline SET POINT (LEVEL \%) & $\gamma$ & $\tau$ & $\lambda$ & $\mathrm{u}_{2}$ \\
\hline 10 & 20 & 1.5 & 0.9 & $6.5868 \mathrm{e}-04$ \\
\hline
\end{tabular}

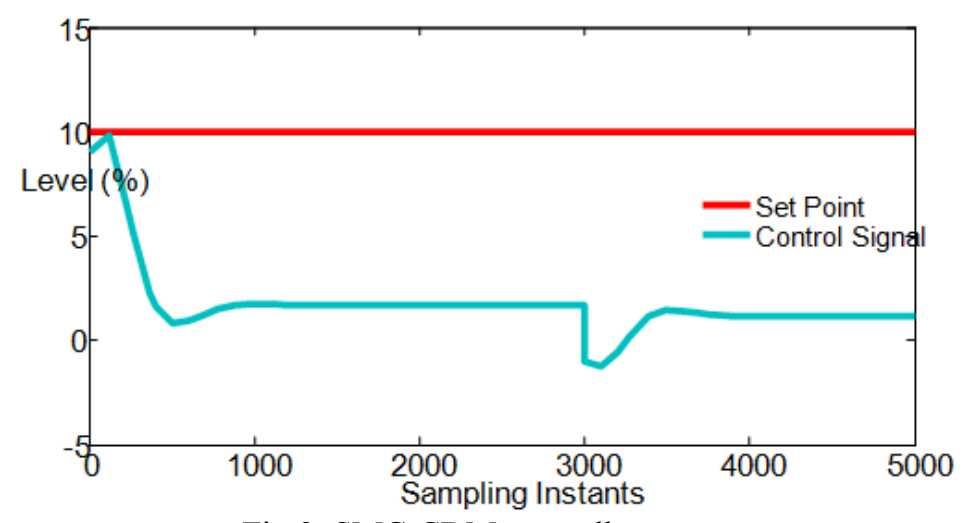

Fig 2. SMC-CDM controller output

Figs. 2 and 3 shows that the controller output of sliding mode controller uses CDM technique and PI controller. And the following figs. 4 shows that the comparison of plant responses with corresponding controller. It can be seen that the time domain and error criteria are well satisfied in SMC-CDM controller. And table 4 provides the comparison of controller performances. 


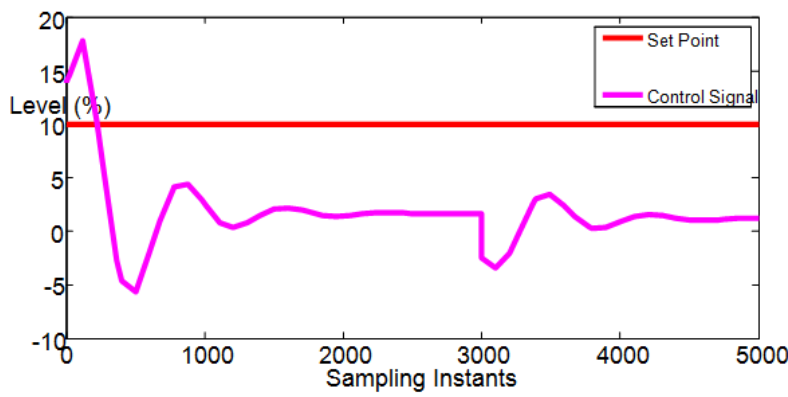

Fig 3. PI controller output

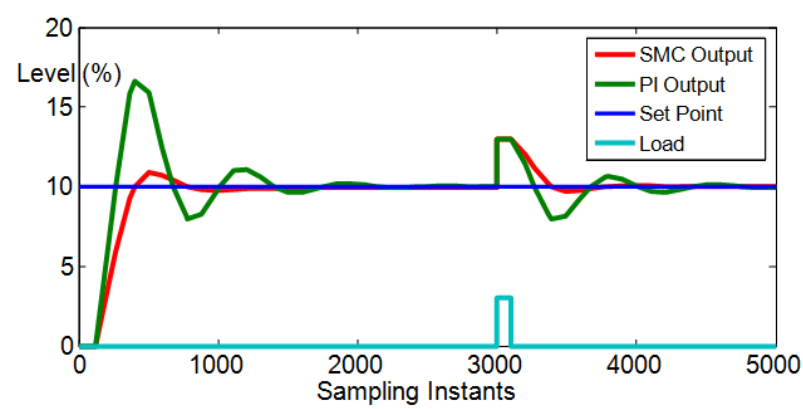

Fig 4. Comparison of servo and regulatory responses with SMC-CDM and PI controller

Table 4. Performance comparison of SMC and PI controller

\begin{tabular}{|l|c|c|l|l|l|l|l|}
\hline CONTROLLER & \multicolumn{2}{|l|}{ LEVEL (\%) } & \multirow{2}{*}{ IAE } & ISE & \multicolumn{2}{l|}{ OVERSHOOT(\%) } & \multicolumn{2}{l|}{ SETTLING TIME (Sec) } \\
\cline { 2 - 6 } & Set point & Load & & & & Set point & Load \\
\hline SMC & 10 & 3 & 3700 & $2.258 \mathrm{e}+04$ & 10.83 & 2135 & 4209 \\
\hline PI & 10 & 3 & 5912 & $2.975 \mathrm{e}+04$ & 16.62 & 2590 & 5000 \\
\hline
\end{tabular}

\section{Real Time Implementation And Responses}

Table 5. Controller settings of SMC-CDM and PI controller(ZN method)

\begin{tabular}{|l|c|c|c|c|c|c|}
\hline LEVEL (CM) & \multicolumn{4}{|c|}{ SMC-CDM } & \multicolumn{2}{c|}{ PI controller } \\
\hline SET POINT & $\gamma$ & $\tau$ & $\Lambda$ & $\mathrm{u}_{2}$ & $k_{p}$ & $k_{i}$ \\
\hline 20 & 20 & 0.3 & 0.9 & 0.0016 & 2.5 & 0.0024 \\
\hline
\end{tabular}

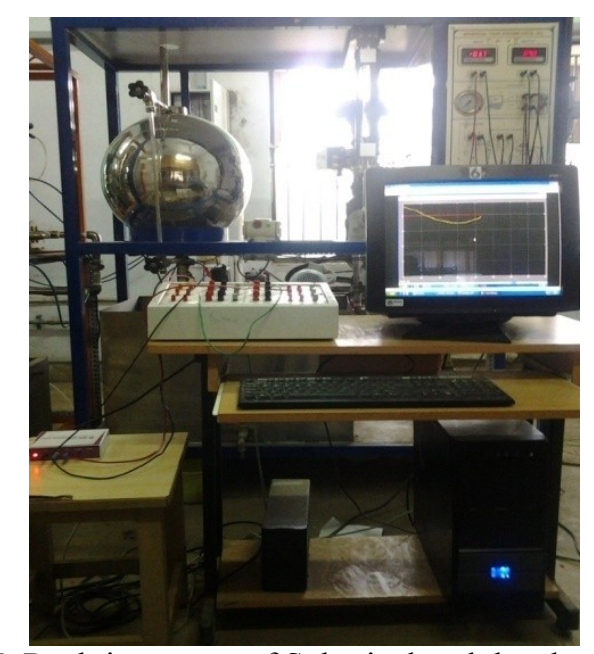

Fig 5. Real time setup of Spherical tank level process

To study the servo and regulatory characteristics of the SMC controller set point change and load is applied to the process. Set point is changed from $20 \%$ of the level to $30 \%$ of the level, and Load is applied at the $2.624 \mathrm{e}+5$ th Sampling Instant. The controller output is shown in the fig (6) and the corresponding plant response is shown in fig (7). 


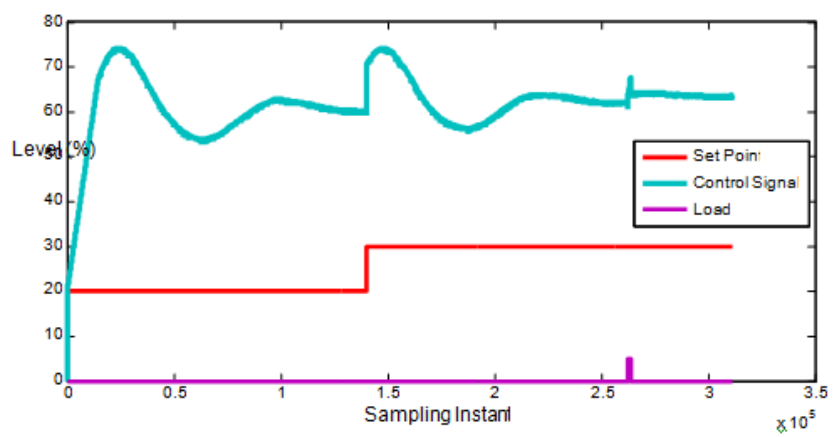

Fig 6. SMC-CDM Controller output in real time

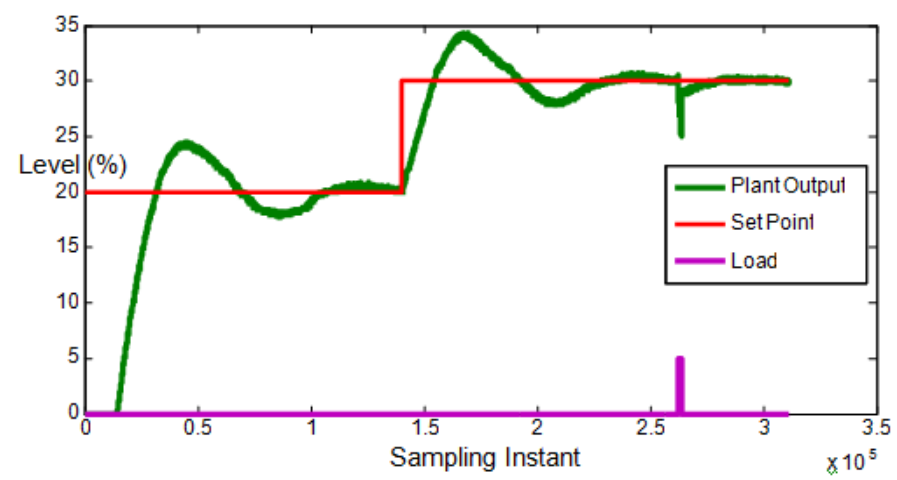

Fig 7. Servo and regulatory responses of plant with SMC-CDM controller in real time

To study the servo and regulatory characteristics of the PI controller set point change and load is applied to the process. Set point is changed from $20 \%$ of the level to $30 \%$ of the level, and Load is applied at the $3.261 \mathrm{e}+5$ th Sampling Instant. The PI controller output is shown in the fig (8) and the corresponding plant output is shown in fig (9). Fig 10 shows that the comparison of SMC-CDM and PI controller performance in closed loop.

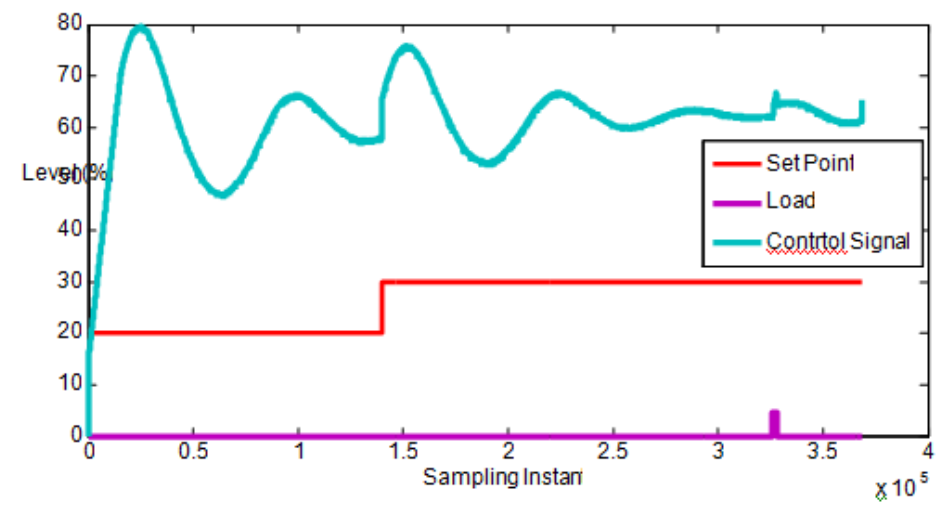

Fig 8. PI controller output in real time

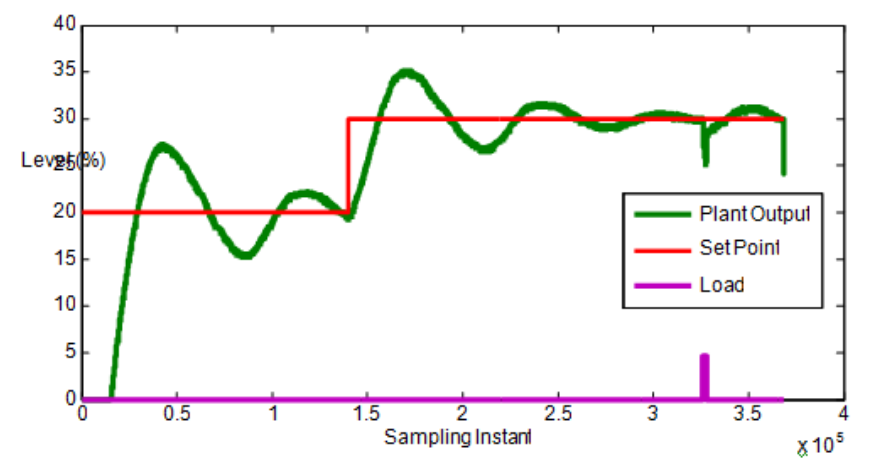

Fig 9. Servo and regulatory response of plant with PI controller in real time 


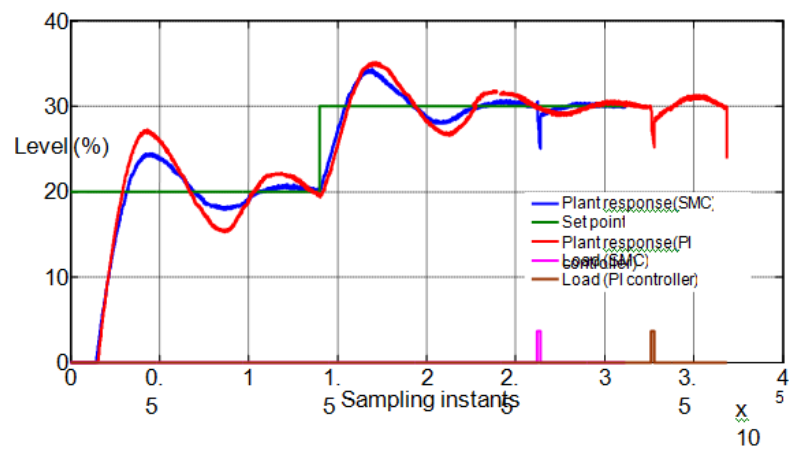

Fig 10. Comparison of plant responses with SMC-CDM and PI controllers

The following table 6 provides the performance comparison of SMC-CDM and PI controller in real time of Spherical tank level process.

Table 6. Performance comparison of SMC-CDM and PI controllers in real time

\begin{tabular}{|l|c|l|l|l|l|l|l|}
\hline CONTROLLER & \multicolumn{2}{|l|}{ LEVEL (\%) } & IAE & ISE & OVERSHOOT(\%) & \multicolumn{2}{|l|}{ SETTLING TIME (Sec) } \\
\cline { 2 - 8 } & $\begin{array}{l}\text { Initial Set } \\
\text { point }\end{array}$ & $\begin{array}{l}\text { Changed set } \\
\text { point }\end{array}$ & & & $\begin{array}{l}\text { Initial Set } \\
\text { point }\end{array}$ & $\begin{array}{l}\text { Changed } \\
\text { set point }\end{array}$ \\
\hline SMC & 20 & 30 & 8902 & $8.913 \mathrm{e}+04$ & 24.48 & $1.374 \mathrm{e}+5$ & $2.546 \mathrm{e}+05$ \\
\hline PI & 20 & 30 & 9749 & $10.53 \mathrm{e}+04$ & 27.62 & $1.397 \mathrm{e}+5$ & $3.208 \mathrm{e}+05$ \\
\hline
\end{tabular}

From the results (Simulation and Real Time) obtained (as shown in this chapter) it is very much clear that the SMC controller performs better than the conventional PI controller in all cases.

\section{Conclusion}

Sliding mode controller for a non-linear process (Spherical Tank) is designed as an algorithm that does not require the derivative of the sliding surfaces is proposed, CDM technique for optimizing controller parameters is implemented and pperformance of designed controller was compared (Both Simulation Real Time). As per the Control algorithm of SMC, it takes more time to find out the optimum values of controller parameters by using Trial and error method, in order to reduce complexity, CDM technique is used to find the parameters. CDM technique is a simple and effective method to find the unknown parameters. It proves that the proposed controller is better one.

\section{REFERENCES}

[1] S. Manabe. Coefficient Diagram Method. Automatic Control in Aerospace, Seoul, Korea. 211-222, 1998.

[2] Mohammad Khalid Khan,. Sarah K Spurgeon."Second order Sliding Mode Control of coupled tanks". 2005, IFAC

[3] Bartolini, G., Ferrara, A., Usai, E., Utkin, V.,. "On multi-input chattering-free-second order sliding mode control". IEEE Trans. on Automatic Control 45(9), 2000, 1711-1718.

[4] Serdar Ethem Hamamci, NusretTan., "Design of PI controllers for achieving time and frequency domain specifications simultaneously". ISA Transactions, Volume 45-4, 2006, pages 529-543

[5] Chang, L.,. “A MIMO sliding control with a second order sliding condition”. In: ASME Winter Annual Meeting. 1990, paper No. 90WA/DSC-5.

[6] G. Bartolini, A. Pisano, E. Usai.,. "Variable Structure Control of Nonlinear Sampled Data Systems by Second Order Sliding Mod es", in "Variable Structure Systems, Sliding Mode and Nonlinear Control", Springer-Verlag, 1999, vol. 247, 43-68.

[7] Satheesh Babu. R, S. Abraham Lincon "Design and Implementation of Model Reference Adaptive Controller using Coefficient Diagram Method for a nonlinear process", IOSR JEEE, 2013, Volume 6, 2320-3331, PP 21-27. 